\title{
VECTOR BUNDLES OVER ORBIT MANIFOLDS
}

\author{
BY \\ DENIS SJERVE
}

1. Introduction. Let $\pi$ denote any finite group, not necessarily abelian, acting freely as diffeomorphisms on some standard sphere $S^{n}$. If $M=M^{n}$ denotes the orbit manifold $S^{n} / \pi$, then it is a compact, connected manifold of dimension $n$ and the quotient map $p: S^{n} \rightarrow M$ is a principal $\pi$-bundle.

If $\xi$ is an orientable real $2 r+1$ bundle over $M$ we may interpret it as the homotopy class of a map $\xi: M \rightarrow$ BSO $(2 r+1)$. Now we ask the following questions:

(1.1) when does $\xi$ pull back to a $2 s-1,1 \leqq s \leqq r$, bundle over $M$ ?

(1.2) when does $\xi$ pull back to a $2 s, 1 \leqq s \leqq r$, bundle over $M$ ?

In other words, we want to study the lifting problem

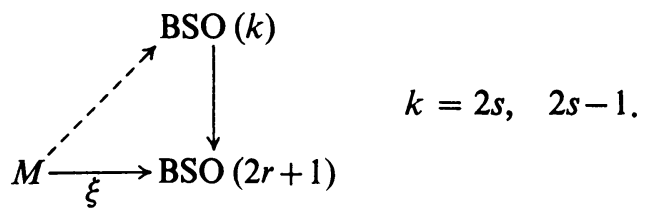

For (1.1) we have the necessary conditions:

(1.3) (i) $\bmod 2$ torsion $P_{s}(\xi)=0, P_{s+1}(\xi)=0, \ldots, P_{r}(\xi)=0$;

(ii) the bundle $p^{*}(\xi)$ over $S^{n}$ pulls back to a $2 s-1$ bundle. (i) follows from the Whitney duality theorem on the integral Pontrjagin classes $P_{i}(\xi)$. Similarly we have the following necessary conditions for (1.2):

(1.4) (i) $\bmod 2$ torsion $P_{s+1}(\xi)=0, \ldots, P_{r}(\xi)=0$;

(ii) there exists $u \in H^{2 s}(M ; Z)$ such that $u^{2}=P_{s}(\xi)$;

(iii) the bundle $p^{*}(\xi)$ over $S^{n}$ pulls back to a $2 s$ bundle.

(ii) is a necessary condition because in $H^{*}(\mathrm{BSO}(2 s) ; Z)$ the Pontrjagin class $P_{s}$ is the square of the Euler class $\chi_{2 s}$.

In this paper we make the following assumption:

(1.5) Hypothesis. $|\pi|=$ the order of $\pi$, is odd.

(1.6) Remark. The case $n=1$ is trivial so we exclude it in all that follows. Also, it is well known that the only group acting freely on an even dimensional sphere is $Z_{2}$. Therefore $n \geqq 3$ is odd and $\pi_{1}(M)=\pi$. Moreover, according to (2.1), every bundle $\xi$ is a spin bundle (i.e., $\left.w_{1}(\xi)=0, w_{2}(\xi)=0\right)$. In (1.3) and (1.4) we may drop the condition on two torsions since $H^{*}(M ; Z)$ has only odd torsion.

Received by the editors March 15, 1968. 
The following theorems give conditions under which (1.3) and (1.4) are also sufficient.

(1.7) THEOREM. Let $\xi$ be any $2 r+1$ bundle over $M$. If $q /|\pi|$ is any prime divisor we assume $q>[n / 2]-2 s+2$. Then $\xi$ pulls back to a $2 s-1$ bundle if (1.3) is true.

(1.8) TheOREM. Again $\xi$ is a $2 r+1$ bundle over $M$. If $q /|\pi|$ is any prime divisor we assume $q>\max ([n / 2]-s+1, s+1)$. Then $\xi$ pulls back to a $2 s$ bundle if $(1.4)$ is true.

(1.9) Remark. These theorems include the cases where the Pontrjagin classes vanish for dimensional reasons (i.e., $s \geqq[n / 4]$ in (1.7) and $s>[n / 4]$ in (1.8)).

(1.10) Remark. If one assumes that the action of $\pi$ on $S^{n}$ is given by some representation of $\pi$ by orthogonal matrices then the possible groups $\pi$ and the possible $n$ have been determined by Vincent (see [11]). In fact, under the restriction that $|\pi|$ is odd, the group $\pi$ is presented by

$$
\sigma^{r}=1, \quad \tau^{s}=1, \quad \tau \sigma \tau^{-1}=\sigma^{t}, \quad \text { where } r>0,|\pi|=r s,
$$

$(t-1) s$ is relatively prime to $r$, and $t^{s} \equiv 1(\bmod r)$. Moreover, any such presented group $\pi$ can act freely and orthogonally on some sphere $S^{n}$. The possible values of $n$ are given by easy arithmetic conditions on $r, s, t$.

In $\S 2$ some necessary lemmas are stated, in $\$ 3$ the proofs are given, and in $\S 4$ applications are given. Finally, I would like to thank Professors Spanier and Thomas of Berkeley and the Forschungsinstitut für Mathematik, ETH.

2. Preliminary lemmas. Let $\boldsymbol{C}_{\pi}$ denote the Serre class of all finite abelian groups whose orders are relatively prime to $|\pi|$ and let $J \in C_{\pi}$ be a generic element. We denote by $\rho$ any of the obvious maps $Z \oplus J \rightarrow Z, Z \oplus J \rightarrow Z_{|\pi|}=$ the cyclic group of order $|\pi|$. Then the following is well known:

(2.1) Lemma. (i) $H^{i}(M ; J)=0$ for $0<i<n$ and $|\pi| \cdot H^{i}(M ; Z)=0$ for $0<i<n$, i.e., the order of every element is a divisor of $|\pi|$;

(ii) $\rho_{*}: H^{i}(M ; Z \oplus J) \cong H^{i}(M ; Z)$ for $0<i<n$ and

$$
\rho_{*}: H^{2 i}(M ; Z) \cong H^{2 i}\left(M ; Z_{|\pi|}\right) \text { for } 0<2 i<n \text {; }
$$

(iii) $H^{2 i+1}(M ; Z)=0$ for $0<2 i+1<n$;

(iv) $p^{*}: H^{n}(M ; J) \cong H^{n}\left(S^{n} ; J\right)$ and $p^{*}: H^{n}(M ; Z \oplus J) \rightarrow H^{n}\left(S^{n} ; Z \oplus J\right)$ is a monomorphism.

For the next lemma let $V_{n, n-m}$ be the Stiefel manifold of $n-m$ frames in $n$-space. Denote by $\bar{n}$ (resp. $\bar{m})$ the greatest (resp. least) odd integer $\leqq n$ (resp. $\geqq m)$ and denote by $S$ the product $S^{2 \bar{m}+1} \times S^{2 \bar{m}+5} \times \cdots \times S^{2 \bar{n}-3}$ where we must include the factor $S^{n-1}$ if $n$ is even and the factor $S^{m}$ if $m$ is even. If $\bar{n}=\bar{m}$ replace $S^{2 \bar{m}+1} \times \cdots \times S^{2 \bar{n}-3}$ by a point. Putting $n_{1}$ equal to the least dimension of all spheres in $S$ we have

(2.2) LEMMA. (i) for all $i, \pi_{i}\left(V_{n, n-m}\right)$ and $\pi_{i}(S)$ are isomorphic mod finite groups.

(ii) Tor $\pi_{i}\left(V_{n, n-m}\right) \in C_{\pi}$ if $i<n_{1}+2 q-3$ for all prime divisors $q /|\pi|$ (recall that $|\pi|$ is odd). 
3. Proofs of Theorems. Recall that we are assuming $|\pi|$ is odd. To prove (1.7) we consider the lifting problem

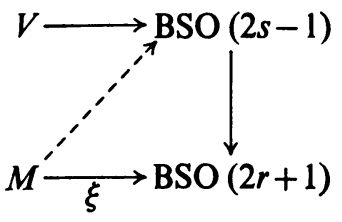

The fiber $V$ is the Stiefel manifold $V_{2 r+1,2 r+1-(2 s-1)}$ so that according to (2.2) we have

(3.2) (i) $\pi_{i}(V)$ and $\pi_{i}\left(S^{4 s-1} \times S^{4 s+3} \times \cdots \times S^{4 s-1}\right)$ are isomorphic mod finite groups;

(ii) Tor $\pi_{i}(V) \in C_{\pi}$ for all $i \leqq n-1$.

(3.3) REMARK. The fiber $V$ is $2 s-2$ connected and therefore is simply connected if $s>1$. If $s=1 V$ is no longer simply connected but we can replace BSO $(k)$ by BSpin $(k)$ without loss of generality (see (1.6)) so that the fiber becomes simply connected.

In the Postnikov resolution of (3.1) we encounter obstructions in the groups $H^{i+1}\left(M ; \pi_{i}(V)\right)$ for $2 s-1 \leqq i \leqq n-1$. But, according to (3.2) and (2.1), these groups are zero for $i \neq 4 s-1,4 s+3, \ldots, 4 s+4 t-1$ and $n-1$, where $t$ is the largest integer such that $4 s+4 i-1 \leqq \min (n-2,4 r-1)$. If $t<0$ there is only one possibly nonzero obstruction (corresponding to $i=n-1$ ). To shorten notation put $\pi_{i}=\pi_{i}(V)$. Then our Postnikov resolution becomes

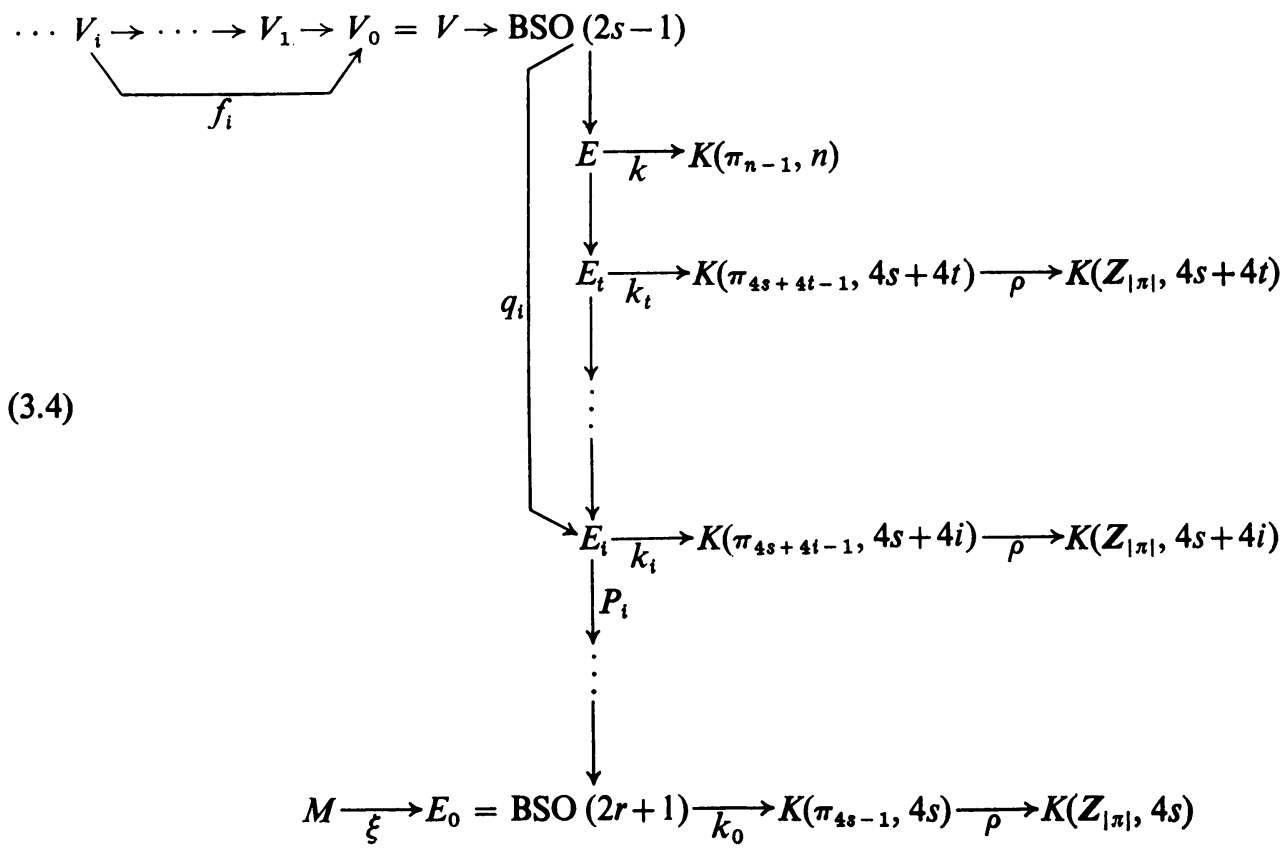


Here the $E_{i}, 0 \leqq i \leqq t$, are the various spaces in the tower and the $k_{i}$ are the corresponding Postnikov invariants. $E$ and $k$ are the top dimensional space and invariant. Notice that we ignore most of the spaces and invariants in the "actual" Postnikov resolution because the corresponding obstructions are zero as remarked above. This makes no essential difference. Finally the spaces $V_{i}$ are the fibers of the $q_{i}$.

By standard theory we know that $\xi$ lifts up to $E_{i+1}$ in (3.4) if, and only if, there exists a lifting $\eta: M \rightarrow E_{i}$ so that the obstruction $\eta^{*}\left(k_{i}\right) \in H^{4 s+4 i}\left(M ; \pi_{4 s+4 i-1}\right)$ is zero. By $(2.1) \eta^{*}\left(k_{i}\right)=0$ if, and only if, $\eta^{*} \rho_{*}\left(k_{i}\right)=0$. The idea is to relate the obstructions in the groups $H^{4 s+4 i}\left(M ; Z_{|\pi|}\right)$ to the Pontrjagin classes $P_{s+i}(\xi), 0 \leqq i \leqq t$, reduced $\bmod |\pi|$. However, suppose for the moment that $\xi$ has been lifted up to $E$ :

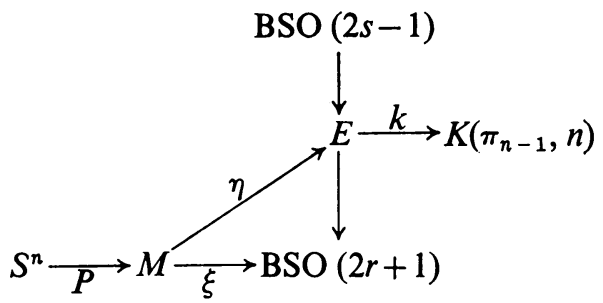

The last obstruction to lifting $\xi$ is $\eta^{*}(k) \in H^{n}\left(M ; \pi_{n-1}\right)$. Now the map $E \rightarrow \mathrm{BSO}(2 r+1)$ is a composite of fibrations whose fibers are Eilenberg-MacLane spaces of type $K(G, j), j \leqq n-2$. Thus it follows that $\pi_{n}(E) \cong \pi_{n}(\mathrm{BSO}(2 r+1))$ via the induced map. In particular $\eta \circ p$ is the unique (up to homotopy) lifting of $\xi \cdot p$ up to $E$. Since $\xi \cdot p$ lifts past $E$ so does $\eta \cdot p$, i.e., $p^{*} \eta^{*}(k)=0$. The homotopy group $\pi_{n-1}$ is either $J$ or $Z \oplus J$, where $J \in C_{\pi}$. But in either case (2.1) implies $p^{*}: H^{n}\left(M ; \pi_{n-1}\right)$ $\rightarrow H^{n}\left(S^{n} ; \pi_{n-1}\right)$ has zero kernel. Thus $\eta^{*}(k)=0$ and $\xi$ lifts up to BSO $(2 s-1)$. In the remainder of the proof we restrict our attention to the $k_{i}$.

Since $H^{*}\left(\mathrm{BSO}(2 r+1) ; Z_{|\pi|}\right)$ is a polynomial algebra on the Pontrjagin classes $P_{1}, \ldots, P_{r}$ we can consider "Pontrjagin classes" $P_{s+i} \in H^{4 s+4 i}\left(E_{i} ; Z_{|\pi|}\right)$ for $s+i \leqq r$. Moreover, we would like to "identify" these "Pontrjagin classes" with the invariants $\rho_{*}\left(k_{i}\right) \in H^{4 s+4 i}\left(E_{i} ; Z_{|\pi|}\right)$ for all $i \leqq t$.

Actually, we have

(3.5) Lemma. $P_{s+i}=0$ in $H^{4 s+4 i}\left(E_{i+1} ; Z_{|\pi|}\right)$ and $=a_{i} \rho_{*}\left(k_{i}\right)$ in $H^{4 s+4 i}\left(E_{i} ; Z_{|\pi|}\right)$, where $a_{i}$ is relatively prime to $|\pi|$.

Proof. By standard Postnikov theory we know that

(3.6) $\pi_{j}\left(V_{i}\right)=0$ for $j<4 s+4 i-1$ and $f_{i^{*}}: \pi_{j}\left(V_{i}\right) \cong \pi_{j}\left(V_{0}\right)$ for $j \geqq 4 s+4 i-1$. By the Serre exact sequence of $q_{i+1}$ it then follows that

$$
q_{i+1}^{*}: H^{j}\left(E_{i+1} ; Z_{|\pi|}\right) \cong H^{j}\left(\mathrm{BSO}(2 s-1) ; Z_{|\pi|}\right)
$$

for $j<4 s+4 i+3$. In particular $P_{s+i}=0$ in $H^{4 s+4 i}\left(E_{i+1} ; Z_{|\pi|}\right)$ since it is zero in 
$H^{4 s+4 i}\left(\right.$ BSO $\left.(2 s-1) ; Z_{|\pi|}\right)$. Note that this implies $P_{s+i}$ is in the image of the transgression of the fibration $q_{i}$ and is therefore a multiple of $\rho_{*}\left(k_{i}\right)$ since

$$
H^{4 s+4 i-1}\left(V_{i} ; Z_{|\pi|}\right) \cong Z_{|\pi|}
$$

is cyclic and $\rho_{*}\left(k_{i}\right)$ transgresses from the fundamental class of $H^{4 s+4 i}\left(E_{i} ; Z_{|\pi|}\right)$. The lemma for $i=0$ follows directly from a theorem of Borel-Hirzebruch (p. 377 of [1]).

Now consider the commutative diagram of fibrations

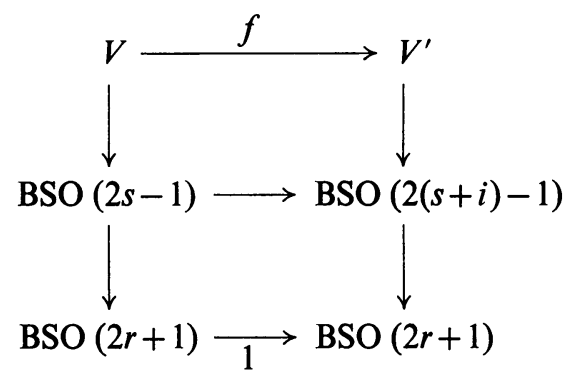

where $s+i \leqq r, V^{\prime}$ is the Stiefel manifold $V_{2 r+1,2 r+1-(2(s+i)-1)}$, and all the maps are standard. We use the notation $E_{j}^{\prime}, k_{j}^{\prime}$ for the right hand fibration in the same way that $E_{j}, k_{j}$ is used for the left hand one. According to Borel-Hirzebruch we have $P_{s+i}=a^{\prime} \rho_{*}\left(k_{o}^{\prime}\right)$ in the resolution of the right hand fibration. By naturality of Postnikov resolutions we have the (homotopy) commutative diagram

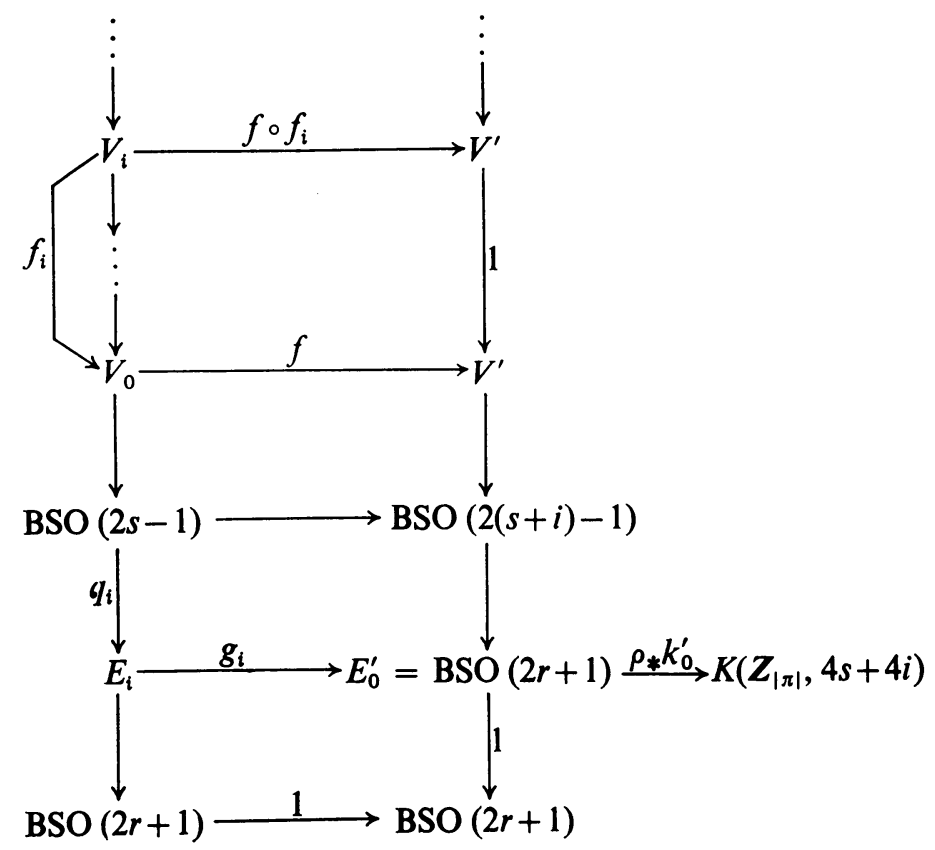


The fiber of $f: V \rightarrow V^{\prime}$ is the Stiefel manifold $V_{2(s+i)-1,2 i}$ and therefore, by (2.2), we have $f_{*}: \pi_{j}(V) \rightarrow \pi_{j}\left(V^{\prime}\right)$ is a $C_{\pi}$ isomorphism if $4 s+2 q-4>j>4(s+i)-4$ for all prime divisors $q$ of $|\pi|$. Also from (2.2) we have $\pi_{j}\left(V^{\prime}\right) \in C_{\pi}$ for $j<4(s+i)-1$. From (3.6) it now follows that $\left(f \circ f_{i}\right)_{*}: \pi_{j}\left(V_{i}\right) \rightarrow \pi_{j}\left(V^{\prime}\right)$ is a $C_{\pi}$ isomorphism if $j<4 s+2 q-4$ for all prime divisors $q$ of $|\pi|$. From the generalized Whitehead theorem (see (3.3)) and the universal coefficient theorem we get

$$
\left(f \circ f_{i}\right)^{*}: H^{j}\left(V^{\prime} ; Z_{|\pi|}\right) \rightarrow H^{j}\left(V_{i} ; Z_{|\pi|}\right)
$$

is an isomorphism if $j<4 s+2 q-5$. The condition $q>[n / 2]-2 s+2$ in (1.7) implies that $4 s+4 t-1<4 s+2 q-5$ for all such $q$. Thus

(3.8) $\left(f \circ f_{i}\right)^{*}: H^{4 s+4 i-1}\left(V^{\prime} ; Z_{|\pi|}\right) \cong H^{4 s+4 i-1}\left(V_{i} ; Z_{|\pi|}\right)$ for all $i \leqq t$. Notice also that both groups are isomorphic to $\boldsymbol{Z}_{|\pi|}$.

Let $\tau_{i}$ be the transgression in $V_{i} \rightarrow$ BSO $(2 s-1) \stackrel{q_{i}}{\rightarrow} E_{i}$ and let $\tau^{\prime}$ be the transgression in $V^{\prime} \rightarrow \mathrm{BSO}(2(s+i)-1) \rightarrow E_{0}^{\prime}$. There are fundamental classes

$$
v_{i} \in H^{4 s+4 i-1}\left(V_{i}, \pi_{4 s+4 i-1}\right)
$$

and $v^{\prime} \in H^{4 s+4 i-1}\left(V^{\prime} ; \pi_{4 s+4 i-1}\left(V^{\prime}\right)\right)$. Moreover, the Postnikov invariants

$$
k_{i} \in H^{4 s+4 i}\left(E_{i} ; \pi_{4 s+4 i-1}\right)
$$

and $k_{0}^{\prime} \in H^{4 s+4 i}\left(E_{0}^{\prime} ; \pi_{4 s+4 i-1}\left(V^{\prime}\right)\right)$ are simply $\tau_{i}\left(v_{i}\right)$ and $\tau^{\prime}\left(v^{\prime}\right)$ resp. By naturality we have $\tau_{i}\left(f \circ f_{i}\right)^{*}\left(\rho_{*}\left(v^{\prime}\right)\right)=g_{i}^{*} \tau^{\prime}\left(\rho_{*}\left(v^{\prime}\right)\right)=g_{i}^{*}\left(\rho_{*} k_{0}^{\prime}\right)$. Now $P_{s+i}=a^{\prime} \rho_{*}\left(k_{0}^{\prime}\right)$, where $a^{\prime}$ is relatively prime to $|\pi|$. Thus, in $H^{4 s+4 i}\left(E_{i} ; Z_{|\pi|}\right)$ we have $P_{s+i}=g_{i}^{*}\left(P_{s+i}\right)=a^{\prime} g_{i}^{*}\left(\rho_{*} k_{0}^{\prime}\right)$ $=\tau_{i}\left(f \circ f_{i}\right)^{*}\left(a^{\prime} \rho_{*}\left(v^{\prime}\right)\right)$. This together with (3.8) and $\rho_{*}\left(k_{i}\right)=\tau_{i}\left(\rho_{*}\left(v_{i}\right)\right)$ concludes the proof of (3.5).

Consider now the situation

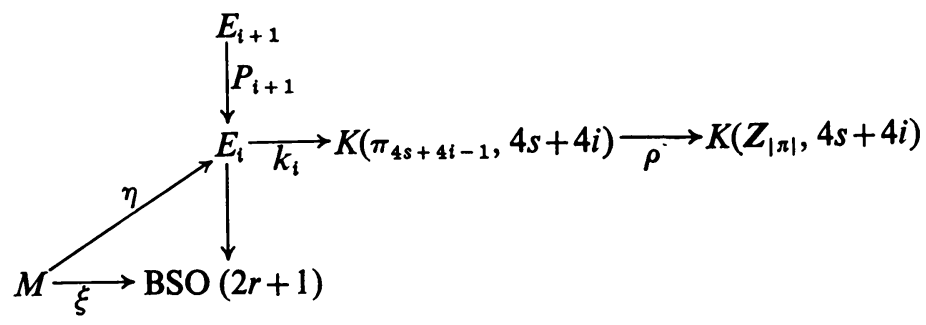

Suppose $\eta: M \rightarrow E_{\mathfrak{i}}$ is some lifting of $\xi$. The $\eta$ will lift up to $E_{i+1}$ if, and only if, $\eta^{*}\left(\rho_{*} k_{i}\right)=0$. By (3.5) and (2.1) this happens if, and only if, $\eta^{*}\left(P_{s+i}\right)=0$. Since $\eta^{*}\left(P_{s+i}\right)=P_{s+i}(\xi)$ and $\rho_{*}: H^{4 s+4 i}(M ; Z) \cong H^{4 s+4 i}\left(M ; Z_{|\pi|}\right)$ this proves (1.7).

To prove (1.8) consider the lifting problem

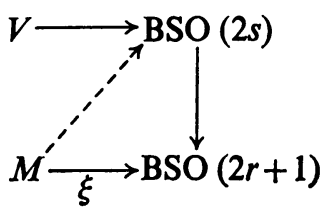


The fiber $V$ is the Stiefel manifold $V_{2 r+1,2 r+1-2 s}$ and therefore we have

(3.10) (i) $\pi_{i}(V)$ and $\pi_{i}\left(S^{2 s} \times S^{4 s+3} \times S^{4 s+7} \times \cdots \times S^{4 r-1}\right)$ are isomorphic mod finite groups;

(ii) Tor $\pi_{i}(V) \in C_{\pi}$ for all $i \leqq n-1$.

In the Postnikov resolution of (3.9) we encounter obstructions in the groups $H^{i+1}\left(M ; \pi_{i}\right)$, where $\pi_{i}=\pi_{i}(V)$, for $i=2 s, 4 s-1,4 s+3,4 s+7, \ldots, 4 s+4 t-5, n-1$, where $t$ is chosen to be the largest integer satisfying $4 s+4 t-5 \leqq \min (n-2,4 r-1)$. The hypothesis of (1.8) allows us to conclude, exactly as in the proof of (3.5), that for $i=4 s+3,4 s+7, \ldots, 4 s+4 t-5$, the obstructions to lifting $\xi$ are the Pontrjagin classes $P_{s+1}(\xi), \ldots, P_{r}(\xi)$. However, this is definitely false for $i=4 s-1$. Our Postnikov resolution therefore becomes

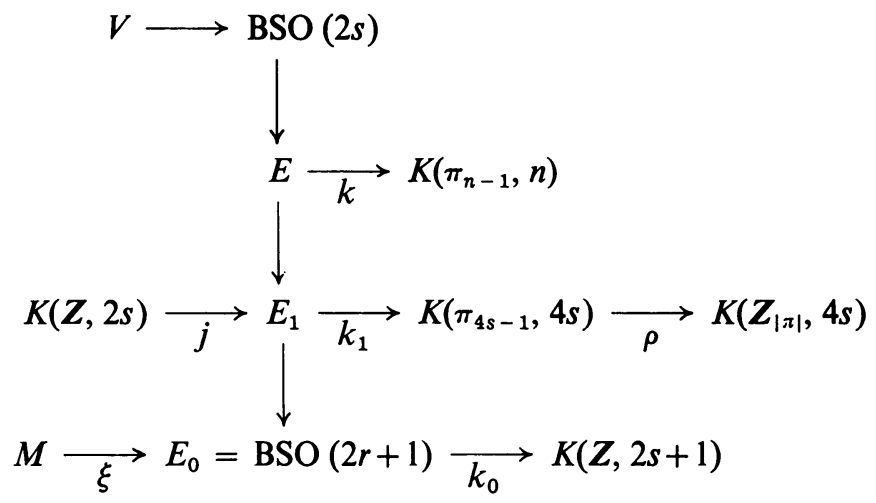

The first obstruction to lifting $\xi$ is $\xi^{*}\left(k_{0}\right) \in H^{2 s+1}(M ; Z)$. By (2.1) this group is zero if $2 s+1 \neq n$. If $2 s+1=n$ the obstruction is known to be the Euler class $\chi(\xi)$ (see [9]). But $\chi(\xi)=\delta w_{2 s}(\xi)$, where $w_{2 s}(\xi)$ is the 2sth Stiefel-Whitney class of $\xi$ and $\delta$ is the coboundary for the coefficient sequence $0 \rightarrow Z \rightarrow Z \rightarrow Z_{2} \rightarrow 0$. Again (2.1) implies $\xi^{*}\left(k_{0}\right)=0$.

The Postnikov invariant $k_{1}$ is treated exactly as in the proof of Theorem B in [8]. It follows from [8] that $j^{*}\left(\rho_{*} k_{1}\right)=\iota^{2}$, where $\iota \in H^{2 s}\left(Z, 2 s ; Z_{|\pi|}\right) \cong Z_{|\pi|}$ is the fundamental class (here is where we use $q>s+1$ for all prime divisors $q$ of $|\pi|$ for then $H^{4 s}\left(Z, 2 s ; Z_{|\pi|}\right) \cong Z_{|\pi|}$ generated by $\left.\imath^{2}\right)$. As in [8] there is a 1-1 correspondence between liftings $\eta: M \rightarrow E_{1}$ and cohomology classes $v \in H^{2 s}(M ; Z)$. Moreover, one has $\eta^{*}\left(\rho_{*} k_{1}\right)=v^{2}+c P_{s}(\xi)$ under this correspondence, where $c$ is any integer so that $4 c+1 \equiv 0(\bmod |\pi|)$. Thus $\xi$ lifts past $E_{1}$ in (3.11) if, and only if, there exists $v \in H^{2 s}(M ; Z)$ satisfying $v^{2}+c P_{s}(\xi)=0$. But this is equivalent to (ii) in (1.4). Finally, the top dimensional obstruction is handled as in the proof of (1.7). This proves (1.8).

4. Applications. Recall that $\pi$ acts freely on $S^{n}$ as diffeomorphisms and $M=S^{n} / \pi$ is the orbit manifold. A theorem of Hirsch (see [2]) states that the manifold $M$ immerses in $\boldsymbol{R}^{n+k}(k \geqq 1)$ if, and only if, the stable normal bundle $\nu$ pulls back to a $k$ bundle. Then (1.3), (1.4), (1.7) and (1.8) imply 
(4.1) TheOREM. (i) Assume $q>[n / 2]-2 s+2$ for all prime divisors $q$ of $|\pi|$. Then $M$ immerses in $R^{n+2 s-1}$ if, and only if, $P_{i}(v)=0$ for $s \leqq i \leqq[n / 4]$.

(ii) $P_{s}(v) \neq 0$ implies $M$ does not immerse in $\boldsymbol{R}^{n+2 s-1}$.

(iii) Assume $q>\max ([n / 2]-s+1, s+1)$ for all prime divisors $q$ of $|\pi|$. Then $M$ immerses in $R^{n+2 s}$ if, and only if, $P_{i}(v)=0$ for $s+1 \leqq i \leqq[n / 4]$ and there exists $u \in H^{2 s}(M ; Z)$ such that $u^{2}=P_{s}(v)$.

(iv) If there is no $u \in H^{2 s}(M ; Z)$ such that $u^{2}=P_{s}(v)$, then $M$ does not immerse in $\boldsymbol{R}^{n+2 s}$.

We can also apply our theorems to the tangent bundle $\tau$ of $M$. Any integer $k \geqq 1$ can be written uniquely in the form $k=2^{4 a+b}(2 c+1)$, where $a, c \geqq 0$ and $0 \leqq b \leqq 3$. Define $\rho(k)=2^{b}+8 a-1$. Also define an $r$-field to be $r$ vector fields everywhere linearly independent. The following theorem is due to Adams, Hurwitz and Radon:

(4.2) THEOREM. $S^{N-1}$ has a $\rho(N)$-field but not a $(\rho(N)+1)$-field.

After a short calculation one easily proves:

(4.3) Lemma. (i) for $N \geqq 0$ we have $\rho(2 N+2) \leqq N+4$ with equality only for $N=3$;

(ii) for $N \neq 3$ we have $\rho(2 N+2) \leqq N+2$ with equality only for $N=1$;

(iii) for $N \neq 0,1,3,7$ we have $\rho(2 N+2) \leqq N$.

First we consider the special cases. Thus we have

(4.4) Theorem. (i) all $M^{3}$ are parallelizable (indeed, every compact orientable 3-manifold is parallelizable);

(ii) all $M^{7}$ admit 4-fields;

(iii) if $3 \nmid|\pi|$, then $M^{7}$ has a 5-field if, and only if, there exists $u \in H^{2}(M ; Z)$ satisfying $P_{1}(M)=u^{2}$;

(iv) if $3 \nmid|\pi|$, then $M^{7}$ is parallelizable if, and only if, $P_{1}(M)=0$;

(v) all $M^{15}$ admit 8-fields.

Let $\rho$ equal the maximum number of everywhere linearly independent vector fields on $S^{n}$ (see (4.2)). Then the general case is

(4.5) TheORem. (i) $n \neq 3,7,15$ implies $M^{n}$ admits 2[ $\left.\rho / 2\right]$-fields (also true for $n=15$ by (v) above). If $\rho$ is even, this is best possible;

(ii) assume $n \neq 3,7,15 ; \rho$ is odd; and $q>(n-\rho) / 2+1$ for all prime divisors $q$ of $|\pi|$. Then $M^{n}$ admits a $\rho$-field.

Expressed somewhat differently these theorems say that $M^{n}$, for $n \neq 7$, has the same maximal number of linearly independent vector fields as the sphere $S^{n}$ provided we assume something about $|\pi|$.

(4.6) Conjecture. $M^{n}$, for $n \neq 7$, has the same number of vector fields as $S^{n}$.

Interesting examples of the orbit manifolds $M$ are the lens spaces. Let $m$ be any odd integer and let $a_{1}, \ldots, a_{n+1}$ be integers relatively prime to $m$. If $\theta$ 
$=\exp (2 \pi(\sqrt{ }-1) / m)$, then $Z_{m}$, the cyclic group presented by: $t, t^{m}=1$, acts on the sphere $S^{2 n+1}$ as follows:

$$
t^{k}\left(z_{1}, \ldots, z_{n+1}\right)=\left(\theta^{k a_{1}} z_{1}, \ldots, \theta^{k a_{n+1}} z_{n+1}\right),
$$

where $\left(z_{1}, \ldots, z_{n+1}\right)$ is a complex $(n+1)$-tuple representing a point of $S^{2 n+1}$. For the orbit manifold we use the notation $L=L^{2 n+1}=L\left(m ; a_{1}, \ldots, a_{n+1}\right)$. The total Pontrjagin class of the tangent bundle $\tau$ is given by (see [7]) $P(\tau)=\left(1+a_{1}^{2} x^{2}\right)$ $\cdots\left(1+a_{n+1}^{2} x^{2}\right)$, where $x \in H^{2}(L ; Z)$ is some class. In particular if all the $a_{i}=a$ this becomes $P(\tau)=\left(1+a^{2} x^{2}\right)^{n+1}$. Since there is no two torsion in $H^{*}(L ; Z)$ the Whitney duality theorem gives

$$
P(\nu)=\sum_{i \geqq 0}(-1)^{i}\left(\begin{array}{c}
n+i \\
i
\end{array}\right) a^{2 i} x^{2 i},
$$

where $\nu$ is the stable normal bundle. Thus

$$
P_{i}(v)=(-1)^{i}\left(\begin{array}{c}
n+i \\
i
\end{array}\right) a^{2 i} x^{2 i}
$$

(4.1) now becomes

(4.7) TheOREM. (i) Assume $q>n-2 s+2$ for all prime divisors $q$ of $m$. Then $L$ immerses in $\boldsymbol{R}^{2 n+2 s}$ if, and only if,

$$
\left(\begin{array}{c}
n+i \\
i
\end{array}\right) \equiv 0 \quad(\bmod m)
$$

for $s \leqq i \leqq[n / 2]$.

(ii)

$$
\left(\begin{array}{c}
n+s \\
s
\end{array}\right) \not \equiv 0 \quad(\bmod m)
$$

implies $L$ does not immerse in $R^{2 n+2 s}$ (here $s \leqq[n / 2]$ ).

(iii) Assume $q>\max (n-s+1, s+1)$ for all prime divisors $q$ of $m$. Then $L$ immerses in $\boldsymbol{R}^{2 n+2 s+1}$ if, and only if,

$$
\left(\begin{array}{c}
n+i \\
i
\end{array}\right) \equiv 0 \quad(\bmod m)
$$

for $s+1 \leqq i \leqq[n / 2]$ and

$$
(-1)^{s}\left(\begin{array}{c}
n+s \\
s
\end{array}\right)
$$

is a quadratic residue $\bmod m$ (here $s \leqq[n / 2]$ ).

(iv) If

$$
(-1)^{s}\left(\begin{array}{c}
n+s \\
s
\end{array}\right)
$$

is not a quadratic residue mod $m$, then $L$ does not immerse in $R^{2 n+2 s+1}($ here $s \leqq[n / 2])$. 
Using (4.7) together with the following theorem of Olum (see [6]) one can give examples of homotopically equivalent lens spaces which have best possible immersions into different dimensions. Such examples have already been given by Kobayashi.

(4.8) THEOREM. $L\left(m ; a_{1}, \ldots, a_{n+1}\right)$ and $L\left(m ; \lambda_{1} a_{1}, \ldots, \lambda_{n+1} a_{n+1}\right)$ are homotopically equivalent if, and only if, there exists an integer $k$ such that $\lambda_{1}, \ldots, \lambda_{n+1}$ $\equiv \pm k^{n+1}(\bmod m)$.

(4.9) ExAmple. Take $n=2$ and $m=5$. Then all the lens spaces $L\left(5 ; a_{1}, a_{2}, a_{3}\right)$ are homotopically equivalent. From (4.1) it easily follows that for $\left(a_{1}, a_{2}, a_{3}\right)$ $=(1,1,1),(1,4,1),(1,1,4),(1,4,4)$ all the corresponding lens spaces do not immerse in $\boldsymbol{R}^{7}$; whereas for any other $\left(a_{1}, a_{2}, a_{3}\right)$ of the form $\left(1, a_{2}, a_{3}\right)$ they do.

(4.10) Example. Take $n=3$ and $m=7$. Then all lens spaces $L^{7}$ are homotopically equivalent. Consider $L=L(7 ; 2,1,1,1)$ and $L^{\prime}=L(7 ; 1,1,1,1)$. Then $P_{1}(L)=0$ and $P_{1}\left(L^{\prime}\right) \neq 0$. Thus $L$ is parallelizable, where $L^{\prime}$ is not.

For references on the immersion problem for lens spaces see [3], [4], [5], [8] and [10].

\section{REFERENCES}

1. A. Borel and F. Hirzebruch, Homogeneous spaces. II, Amer. J. Math. 81 (1959), 315-382.

2. Morris W. Hirsch, Immersions of manifolds, Trans. Amer. Math. Soc. 93 (1959), 242-267.

3. T. Kambe, The structure of $K_{\mathrm{A}}$ rings of the lens space and their application, J. Math. Soc. Japan 18 (1966), 135-146.

4. T. Kobayashi, Non immersion theorems for lens spaces, J. Math. Kyoto Univ. 6 (1966), 91-108.

5. T. Kobayashi and R. Nakagawa, Non imbeddability of lens spaces mod 3, J. Math. Kyoto Univ. 5 (1966), 313-324.

6. P. Olum, Mappings of manifolds and the notion of degree, Ann. of Math. (2) 58 (1953), 458-480.

7. R. H. Szczarba, On tangent bundles of fiber spaces and quotient spaces, Amer. J. Math. 86 (1964), 685-697.

8. D. Sjerve, Geometric dimension of vector bundles over lens spaces, Trans. Amer. Math. Soc. 134 (1968), 545-558.

9. Emery Thomas, Seminar on fiber spaces, Vol. 13, Lecture notes in math., Springer-Verlag, Berlin, 1966.

10. Fuichi Uchida, Immersions of lens spaces, Tôhoku Math. J. 18 (1966), 393-397.

11. Georges Vincent, Les groupes linéaires finis sans points fixes, Comment. Math. Helv. 20 (1947), 117-171.

\section{FORSCHUNGSINSTITUT FÜR MATHEMATIK,} ZÜRICH, SWITZERLAND 